\title{
Polypharmacy Is Associated With Accelerated Deterioration of Renal Function in Cardiovascular Outpatients
}

\author{
Jun-ichi Sakamoto a, b, f, Toshiyuki Shikatac, Satoyasu Ito ${ }^{\mathrm{a}, \mathrm{d}}$, Takeshi Kimura ${ }^{\mathrm{b}}$, Kojiro Takamoto ${ }^{\mathrm{a}, \mathrm{c}}$, \\ Eri Manabe ${ }^{\mathrm{e}}$, Masanori Asakura ${ }^{\mathrm{e}}$, Masaharu Ishihara ${ }^{\mathrm{e}}$, Takeshi Tsujino ${ }^{\mathrm{a}, \mathrm{d}, \mathrm{e}}$
}

\begin{abstract}
Background: Polypharmacy is associated with poor prognosis of patients with various diseases. However, it has not been precisely addressed how polypharmacy affects the clinical characteristics of the cardiovascular outpatients. The aim of this study is to search for the clinical characteristics related to the number of prescribed drugs in the cardiovascular outpatients. Also, we examine whether the number of the prescribed drugs affects the worsening of renal function.

Methods: This retrospective study was conducted using the data of 259 continuous cardiovascular outpatients who were examined complete blood count $(\mathrm{CBC})$ and serum creatinine.

Results: In the univariate analysis, the number of prescribed drugs were associated with the number of cardiovascular diseases or their risk factors, age, white blood cells, platelet, body mass index, anemia, and chronic kidney disease stage $3 \mathrm{~b}$ or higher. In the multivariable analysis, independent variables that significantly correlated with the number of prescribed drugs were the number of cardiovascular diseases or their risk factors, anemia, and chronic kidney disease stage $3 \mathrm{~b}$ or higher. Among 259 patients, 208 patients received follow-up examination of serum creatinine. The number of prescribed drugs was the only factor that was associated with accelerated deterioration of renal function.
\end{abstract}

Conclusions: Polypharmacy is associated not only with poor renal function but with accelerated deterioration of renal function. Polypharmacy may be causally related with renal dysfunction.

Manuscript submitted November 22, 2019, accepted December 21, 2019

${ }^{a}$ Graduate School of Pharmacy, Hyogo University of Health Sciences, Kobe, Japan

${ }^{b}$ Department of Pharmacy, Hyogo College of Medicine Hospital, Nishinomiya, Japan

${ }^{\mathrm{c} D e p a r t m e n t}$ of Pharmacy, Hyogo College of Medicine Sasayama Medical Center, Sasayama, Japan

${ }^{\mathrm{d} D e p a r t m e n t}$ of Pharmacy, School of Pharmacy, Hyogo University of Health Sciences, Kobe, Japan

e Cardiovascular Division and Division of Coronary Heart Disease, Department of Internal Medicine, Hyogo College of Medicine, Nishinomiya, Japan

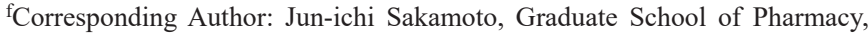
Hyogo University of Health Sciences, 1-3-6 Minatojima, Chuo-ku, Kobe, 6508530, Japan. Email: jun7.3xyz@gmail.com

doi: https://doi.org/10.14740/cr991
Keywords: Polypharmacy; Renal function; Anemia; Chronic kidney disease

\section{Introduction}

The older population is increasing rapidly in developed countries because of declining fertility and increasing life expectancy [1]. Generally, the older population is tended to suffer from multiple chronic diseases such as heart failure, angina pectoris, peripheral artery disease, hypertension, arthritis, dyslipidemia, stroke and diabetes, which require multiple medications for proper treatment [2-4]. It has been reported that about $80 \%$ of older population aged 65 or older have at least one chronic disease, and about 50\% have at least two [5].

Multiple medication use, widely referred to as polypharmacy, is clinical issue in older population [6]. There is no standard cut point with regard to multiple medications, but polypharmacy is usually referred to five or more medications in Japan according to Guidelines for Medical Treatment and its Safety in the Elderly 2015 [7]. Polypharmacy is associated with poor adherence, drug-drug interactions, medication errors, increased health care costs, reduced functional ability, and increased risk of geriatric syndromes, such as cognitive impairment, falls, malnutrition, urinary incontinence [6, 8-13]. Thus, polypharmacy is accounting for a significant percentage of potentially preventable emergency room visits and hospitalization [14]. However, it is still unknown whether polypharmacy causes poor prognosis. Polypharmacy may be just a marker of multiple comorbidities, and multiple comorbidities are the real causes of poor prognosis.

Cardiovascular diseases are common in the older population. The patients with cardiovascular diseases often have various complications and comorbidities and tend to receive multiple medications. Moreover, many evidence-based clinical practice guidelines are created and available nowadays for cardiovascular diseases because many large-scale clinical trials have been conducted in this field. The guideline is basically made for each one disease. If all of the available clinical practice guidelines for each disease were to be applied, the patients with multiple comorbidities could easily fall into polypharmacy. Thus, proper management of polypharmacy is an important clinical issue in patients with cardiovascular diseases. However, there are few reports about the clinical factors or laboratory findings associated with multiple medications in 
patients with cardiovascular diseases. The aim of this study is to search for the clinical characteristics related to the number of prescribed drugs in the cardiovascular outpatients. Also, we examine whether the number of the prescribed drugs affects the worsening of renal function.

\section{Materials and Methods}

\section{Population and data collection}

This retrospective study was conducted using the data of continuous outpatients visiting the Cardiovascular Division, Department of Internal Medicine, Hyogo College of Medicine Hospital from February 2011 to October 2012 for the treatment of cardiovascular disease (ischemic heart disease, heart failure, peripheral artery disease, cerebrovascular disease, cardiomyopathy, and arrhythmia) or risk factors of cardiovascular disease (hypertension, dyslipidemia, diabetes mellitus, and hyperuricemia), and who were examined complete blood count (CBC) and serum creatinine. Patients with malignant tumor, inflammatory diseases, hemorrhagic disease, hemodialysis, blood diseases, collagen disease or the patients who use steroid, erythropoietin, iron preparation were excluded from the investigation. We collected patient data such as demographics, comorbidity, and medication in their clinical records. Estimated glomerular filtration rate (eGFR) was calculated with Japanese equation: eGFR $\left(\mathrm{mL} / \mathrm{min} / 1.73 \mathrm{~m}^{2}\right)=$ $194 \times$ serum creatinine ${ }^{-1.094} \times$ age $^{-0.287}(\times 0.739$ if female $)$. The changing rate of eGFR was evaluated in patients who received another eGFR examination more than 1 year later after the basal eGFR examination. The changing rate of eGFR was calculated by dividing the difference of eGFR between two examinations by the number of years between the two examinations. The comorbidities that we counted as the number of cardiovascular diseases or their risk factors were hypertension, diabetes mellitus, dyslipidemia, heart failure, ischemic heart disease, stroke, peripheral artery disease, and atrial fibrillation. Hypertension was defined as systolic blood pressure (SBP) $\geq 140 \mathrm{~mm} \mathrm{Hg}$, diastolic blood pressure $(\mathrm{DBP}) \geq 90 \mathrm{~mm} \mathrm{Hg}$, or use of anti-hypertensive medication. Diabetes mellitus was defined as fasting plasma glucose $\geq 126$ $\mathrm{mg} / \mathrm{dL}$, hemoglobin A1c $(\mathrm{HbA} 1 \mathrm{c}) \geq 6.5 \%$, or use of anti-diabetic medication. Dyslipidemia was defined as low-density lipoprotein cholesterol $\geq 140 \mathrm{mg} / \mathrm{dL}$, high-density lipoprotein cholesterol < $40 \mathrm{mg} / \mathrm{dL}$, triglyceride $\geq 150 \mathrm{mg} / \mathrm{dL}$, or use of anti-dyslipidemia medication. Anemia was defined according to WHO criteria (for men: less than $13 \mathrm{~g} / \mathrm{dL}$ of hemoglobin, for women: less than 12 $\mathrm{g} / \mathrm{dL}$ of hemoglobin). Chronic kidney disease (CKD) stage was defined according to KDIGO 2012 clinical practice guideline for the evaluation and management of CKD [15].

\section{Statistical analysis}

All statistical analyses were performed with EZR on R Commander Version 1.35 (Saitama Medical Center, Jichi Medical University, Saitama, Japan) [16], which is a graphical user interface for R (The R Foundation for Statistical Computing, Vienna, Austria). The data are presented as mean \pm standard deviation
(SD) for normally distributed variables, median (interquartile rang) for not normally distributed variables, or the number (percentage) for categorical variables. Differences in categorical variables between groups were assessed using Fisher's exact test. Differences in continuous variables between groups were assessed using unpaired Student's $t$ - test or one-way analysis of variance (ANOVA) followed by Tukey's post hoc test for normally distributed variables, or Kruskal-Wallis's test followed by Steel-Dwass's post hoc test for not normally distributed variables. Regarding continuous variables, the correlation between each variable was examined using Spearman's rank correlation coefficient. The factors which were independently associated with the number of drugs were analyzed using linear regression analysis. Factors with $\mathrm{P}<0.10$ in Spearman's rank correlation coefficient were selected as independent factors in multivariate analysis.

\section{Ethics approval}

This investigation was approved by the Ethics Committee of Hyogo College of Medicine Hospital (\#1900) and the Ethics Committee of Hyogo University of Health Sciences (\#15009).

\section{Results}

\section{Study population}

We screened 634 patients, and 259 patients were enrolled in this study. Characteristics of the study population are shown in Table 1. The patients were categorized as follow: the patients prescribed less than five drugs were categorized as the non-polypharmacy group $(\mathrm{N}=68,26.3 \%)$, five to nine drugs were categorized as the polypharmacy group $(\mathrm{N}=132,51 \%)$, and more than 10 drugs were categorized as the hyper-polypharmacy group $(\mathrm{N}=59,22.8 \%)$. The median of the number of prescribed drugs in whole study population was seven, and $73.8 \%$ of patients were prescribed more than five drugs. The age of total population was $70.3 \pm 10.8$ years, and the older population have tendency to be prescribed more drugs. eGFR was lower in the polypharmacy and the hyper-polypharmacy group than in the non-polypharmacy group. Furthermore, the numbers of cardiovascular diseases or their risk factors were increased in the polypharmacy and the hyper-polypharmacy groups. As the number of the prescribed drugs increased, red blood cells $(\mathrm{RBC})$, hemoglobin $(\mathrm{Hb})$ and hematocrit $(\mathrm{Ht})$ decreased. The prevalence of anemia was also increased in the polypharmacy and the hyper-polypharmacy groups. Drugs administered in each category of polypharmacy are shown in Table 2. The proportion of each prescribed drug was higher in the polypharmacy and the hyper-polypharmacy groups except for cardiac stimulant, calcium-channel blocker and $\mathrm{H}_{2}$ blocker.

\section{The number of the prescribed drugs in the patients with each comorbidity}

The number of prescribed drugs increased when the patients 
Table 1. Clinical Characteristics

\begin{tabular}{|c|c|c|c|c|c|}
\hline & \multicolumn{4}{|c|}{ Classification of polypharmacy } & \multirow{2}{*}{ P value } \\
\hline & All & non-PP $(0$ - 4) & $\mathrm{PP}(5$ - 9) & hyper-PP $(\geq 10)$ & \\
\hline $\mathrm{N}$ & 259 & $68(26.25)$ & $132(50.97)$ & $59(22.78)$ & \\
\hline Male gender $(\%)$ & $173(66.8)$ & $38(55.9)$ & $95(72.0)$ & $40(67.8)$ & 0.075 \\
\hline BMI $\left(\mathrm{kg} / \mathrm{m}^{2}\right)$ & $24.0 \pm 3.9$ & $23.2 \pm 3.7$ & $24.0 \pm 3.9$ & $24.8 \pm 3.8$ & 0.069 \\
\hline $\operatorname{RBC}\left(10^{4} / \mu \mathrm{L}\right)$ & $441 \pm 63$ & $455 \pm 47$ & $444 \pm 61$ & $417 \pm 78^{b, c}$ & 0.002 \\
\hline $\mathrm{Hb}(\mathrm{g} / \mathrm{dL})$ & $13.5 \pm 1.8$ & $14.1 \pm 1.4$ & $13.6 \pm 1.9$ & $12.8 \pm 1.8^{\mathrm{b}, \mathrm{c}}$ & $<0.001$ \\
\hline Ht $(\%)$ & $40.9 \pm 5.0$ & $42.3 \pm 3.8$ & $41.1 \pm 5.3$ & $39.1 \pm 5.2^{\mathrm{b}, \mathrm{c}}$ & 0.002 \\
\hline RDW-CV & $13.6(13.0,14.1)$ & $13.3(12.8,13.7)$ & $13.7(13.1,14.1)^{\mathrm{a}}$ & $13.7(13.1,14.8)^{b}$ & $<0.001$ \\
\hline Number of CVDs or their risk factors & $3(2,4)$ & $2(1,2)$ & $3(2,4)^{\mathrm{a}}$ & $4(2,5)^{b, c}$ & $<0.001$ \\
\hline \multicolumn{6}{|l|}{ CVD or its risk factor } \\
\hline Atrial fibrillation (\%) & $47(18.1)$ & $10(14.7)$ & $22(16.7)$ & $15(25.4)$ & 0.262 \\
\hline Diabetes mellitus (\%) & $98(37.8)$ & $12(17.6)$ & $52(39.4)^{\mathrm{a}}$ & $34(57.6)^{\mathrm{b}, \mathrm{c}}$ & $<0.001$ \\
\hline Dyslipidemia (\%) & $150(57.9)$ & $32(47.1)$ & $81(61.4)$ & $37(62.7)$ & 0.114 \\
\hline Heart failure (\%) & $56(21.6)$ & $7(10.3)$ & $24(18.2)$ & $25(42.4)^{\mathrm{b}, \mathrm{c}}$ & $<0.001$ \\
\hline Hypertension (\%) & $184(71.0)$ & $43(63.2)$ & $94(71.2)$ & $47(79.7)$ & 0.127 \\
\hline IHD (\%) & $129(49.8)$ & $20(29.4)$ & $71(53.8)^{\mathrm{a}}$ & $38(64.4)^{\mathrm{b}}$ & $<0.001$ \\
\hline PAD (\%) & $24(9.3)$ & $1(1.5)$ & $10(7.6)$ & $13(22.0)^{\mathrm{b}, \mathrm{c}}$ & $<0.001$ \\
\hline
\end{tabular}

The data are presented as mean \pm SD for normally distributed variables, median (first quartile, third quartile) for not normally distributed variables, or the number (percentage) for categorical variables. Differences in categorical variables between groups were assessed using Fisher's exact test followed by Holm's post hoc test. Differences in continuous variables between groups were assessed using one-way analysis of variance (ANOVA) followed by Tukey's post hoc test for normally distributed variables, or Kruskal-Wallis's test followed by Steel-Dwass's post hoc test for not normally distributed variables. BMI: body mass index; Cr: serum creatinine; CV: cardiovascular; CVD: cardiovascular disease; eGFR: estimated glomerular filtration rate; Hb: hemoglobin; Ht: hematocrit; IHD: ischemic heart disease; PAD: peripheral artery disease; PIt: platelet; PP: polypharmacy; RBC: red blood cells; RDW-CV: red blood cell distribution width-coefficient of variation; WBC: white blood cell. aP $<0.05$, non-PP vs. PP; bP $<0.05$, non-PP vs. hyper-PP; ${ }^{c} P<0.05$, PP vs. hyper-PP.

suffered from each comorbidity, especially when they had peripheral arterial disease or heart failure (Fig. 1). The numbers of the prescribed drugs also increased in the patients with CKD stage $3 \mathrm{~b}$ or higher $\left(\mathrm{eGFR}<45 \mathrm{~mL} / \mathrm{min} / 1.73 \mathrm{~m}^{2}\right)$, which is associated with higher mortality from cardiovascular diseases than CKD stage 1 or 2 [15]. The patients with anemia received more medications than those without anemia (Fig. 1).

\section{The factors which correlated with the number of the pre- scribed drugs}

In univariate analyses, the number of the prescribed drugs was positively correlated with the number of cardiovascular diseases or their risk factors, age, white blood cells (WBC) and body mass index (BMI), and negatively correlated with eGFR, $\mathrm{Hb}$ and platelet (Table 3). In the multivariable analysis, independent variables that significantly correlated with the number of prescribed drugs were anemia, CKD stage $3 \mathrm{~b}$ or higher, and the number of cardiovascular diseases or their risk factors (Table 4).

\section{The number of the prescribed drugs affects the change of the renal function}

To explore the causal relationship of the number of the prescribed drugs to the renal function, we examined the effect of the number of the prescribed drugs on the changing rate of eGFR in patients who received another eGFR examination more than 1 year later after the basal eGFR examination $(\mathrm{N}$ $=208)$. This analysis revealed that larger number of the drugs was associated with faster declining of eGFR (Table 5). Except 
Table 2. Drugs Administered in Each Category of Polypharmacy

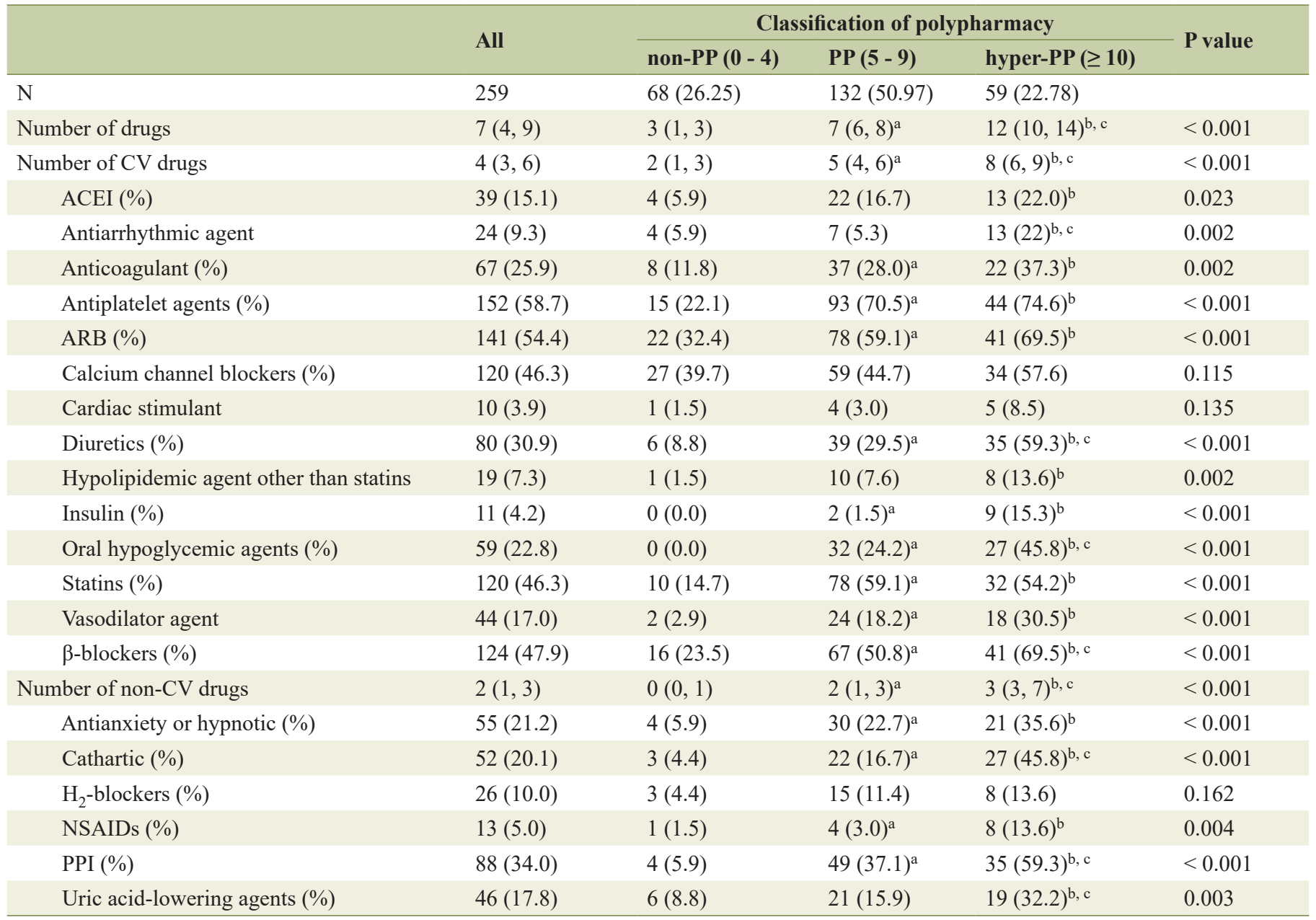

The data are presented as median (first quartile, third quartile) for not normally distributed variables, or the number (percentage) for categorical variables. Differences in categorical variables between groups were assessed using Fisher's exact test followed by Holm's post hoc test. Differences in continuous variables between groups were assessed using Kruskal-Wallis's test followed by Steel-Dwass's post hoc test for not normally distributed variables. ACEI: angiotensin-converting enzyme inhibitor; ARB: angiotensin receptor blocker; CV: cardiovascular; $\mathrm{H}_{2}$-blocker: histamine type 2 receptor blocker; NSAID: nonsteroidal anti- inflammatory drug; PP: polypharmacy; PPI: proton pump inhibitor; statins: HMG-CoA reductase inhibitor. aP < 0.05 , non-PP vs. PP; ${ }^{b} P<0.05$, non-PP vs. hyper-PP; ${ }^{c} P<0.05$, PP vs. hyper-PP.

for the number of prescribed drugs, no clinical factors or laboratory data correlated with the changing rate of eGFR (data not shown). These results suggest that polypharmacy might worsen the prognosis of cardiovascular outpatients by deteriorating the renal function.

\section{Discussion}

We searched for the clinical factors associated with polypharmacy in the cardiovascular outpatients. We found that polypharmacy is associated not only with multiple comorbidities but also with poor renal function and anemia. Silverberg et al have proposed the idea of the cardio-renal anemia syndrome [17], which emphasizes that cardiovascular diseases, CKD, and anemia influence each other and form a vicious circle. For example, the poor renal function is an independent risk factor of car- diovascular disease such as hypertension [18], atrial fibrillation $[19,20]$, heart failure [21], stroke [19], and acute myocardial infarction [22]. Anemia is also a risk factor of the cardiovascular disease [20, 21, 22-26]. Impaired renal function and anemia are not only the risk and prognostic factors of cardiovascular diseases but also the index of severity of cardiovascular diseases $[19,20,22,25]$. In our survey, along with the number of the cardiovascular diseases or their risk factors, CKD stage $3 b$ or higher, and anemia were independently correlated to polypharmacy in multivariable analysis. This finding suggests that polypharmacy is not only the marker of multiple comorbidities, but also a marker of the severity of each comorbidity. It is necessary to consider the effect of renal function and anemia when we analyze the effect of polypharmacy on the prognosis.

Our data have shown that polypharmacy correlated with baseline of eGFR. However, it is unknown whether the polypharmacy deteriorates renal function, or multiple comorbidities 


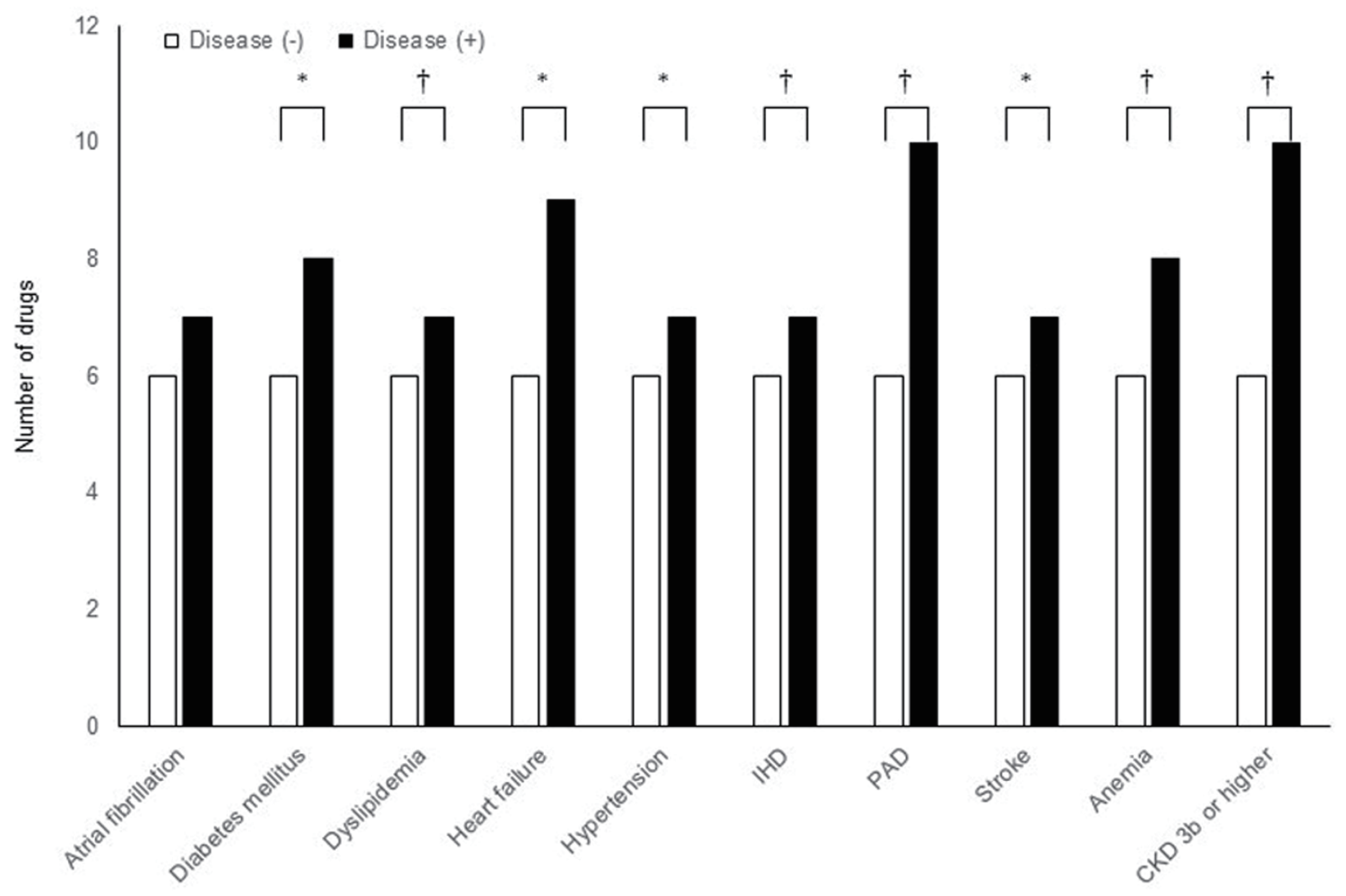

Figure 1. The numbers of drugs prescribed for the patients with each comorbidity as the median of each group. ${ }^{*} P<0.05, \dagger P<$ 0.01 in Mann-Whitney U-test.

cause decrease in renal function. When we examined the factors which correlated with the changing rate of eGFR in the patients who received another eGFR examination more than 1 year later after the basal eGFR examination, the number of the prescribed drugs was the only factor which correlated with the changing rate of eGFR. The changing rate of eGFR did not correlate with comorbidities or other clinical backgrounds. Our data suggested that polypharmacy not only reflects the number and severity of comorbidities but causes the decrease in renal function. Polypharmacy was associated with the use of medications harmful for renal function, such as nonsteroi-

Table 3. Univariate Analyses of Factors Associated With the Number of Drugs

\begin{tabular}{lll}
\hline Factor & $\rho$ & P value \\
\hline Number of CVDs or their risk factors & 0.484 & $<0.001$ \\
eGFR $\left(\mathrm{mL} / \mathrm{min} / 1.73 \mathrm{~m}^{2}\right)$ & -0.336 & $<0.001$ \\
$\mathrm{Hb}(\mathrm{g} / \mathrm{dL})$ & -0.217 & $<0.001$ \\
$\mathrm{Plt}\left(10^{4} / \mu \mathrm{L}\right)$ & -0.153 & 0.014 \\
Age $(\mathrm{years})$ & 0.151 & 0.015 \\
WBC $\left(10^{2} / \mu \mathrm{L}\right)$ & 0.143 & 0.021 \\
$\mathrm{BMI}\left(\mathrm{kg} / \mathrm{m}^{2}\right)$ & 0.127 & 0.041 \\
\hline
\end{tabular}

Correlations between each variable were examined using Spearman's rank correlation coefficient rho $(\rho)$. BMI: body mass index; CVD: cardiovascular disease; eGFR: estimated glomerular filtration rate; $\mathrm{Hb}$ : hemoglobin; PIt: platelet; WBC: white blood cell. dal anti-inflammatory drugs (NSAIDs) or diuretics. However, no single drug was associated with faster deterioration in renal function. Combination of potential nephrotoxic drugs may have induced the accelerated deterioration in renal function.

This study has several limitations. First, the number of patients is rather small. Second, study population is heterogeneous, and many confounding factors affect the results. However, the study population reflects the "real world" of cardiovascular outpatients, and our results may make the physicians realize the impact of polypharmacy in a clinical setting.

Table 4. Multivariate Analysis of Factors Which Were Independently Associated With the Number of Drugs

\begin{tabular}{lll} 
Factor & $\boldsymbol{\beta}$ & P value \\
\hline Number of CVDs or their risk factors & 1.033 & $<0.001$ \\
Anemia & 1.722 & $<0.001$ \\
CKD stage 3b or higher & 1.923 & 0.002 \\
WBC $\left(10^{2} / \mu \mathrm{L}\right)$ & 0.022 & 0.081 \\
Plt $\left(10^{4} / \mu \mathrm{L}\right)$ & -0.049 & 0.229 \\
Male & 0.504 & 0.244 \\
BMI $\left(\mathrm{kg} / \mathrm{m}^{2}\right)$ & 0.025 & 0.638 \\
Age $($ years $)$ & -0.009 & 0.672 \\
\hline
\end{tabular}

Factors which were independently associated with the number of drugs were examined using linear regression analysis. CVD: cardiovascular disease; CKD: chronic kidney disease; WBC: white blood cell; PIt: platelet; BMI: body mass index. 
Table 5. The Factors Which Correlated With the Changing Rate of eGFR

\begin{tabular}{lll} 
Factor & $\boldsymbol{\rho}$ & P value \\
\hline Number of prescribed drugs & 0.151 & 0.030 \\
Age & 0.087 & 0.213 \\
$\mathrm{Plt}$ & -0.072 & 0.298 \\
$\mathrm{Hb}$ & -0.065 & 0.353 \\
$\mathrm{eGFR}$ & -0.061 & 0.378 \\
Number of CVDs or their risk factors & 0.050 & 0.473 \\
WBC & -0.048 & 0.494 \\
BMI & 0.024 & 0.728 \\
\hline
\end{tabular}

The factors which correlated with the changing rate of eGFR were examined using Spearman's rank correlation coefficient. eGFR: estimated glomerular filtration rate; PIt: platelet; $\mathrm{Hb}$ : hemoglobin; CVD: cardiovascular disease; WBC: white blood cell; BMI: body mass index.

\section{Conclusions}

In conclusion, polypharmacy is associated with the number of cardiovascular diseases or their risk factors, renal dysfunction and anemia. Polypharmacy also predicts the accelerated worsening of renal function.

\section{Acknowledgments}

None to declare.

\section{Financial Disclosure}

None to declare.

\section{Conflict of Interest}

None to declare.

\section{Informed Consent}

Informed consent was obtained in the form of opt-out on the web-site of the Cardiovascular Division and Division of Coronary Heart Disease, Department of Internal Medicine, Hyogo College of Medicine.

\section{Author Contributions}

Jun-ichi Sakamoto and Takeshi Tsujino contributed to the conception and design of the work; Jun-ichi Sakamoto, Toshiyuki Shikata, Kojiro Takamoto, Eri Manabe, Satoyasu Ito, and Masanori Asakura contributed to the literature search, data analysis for the work, and drafting the manuscript; Takeshi Kimura and
Masaharu Ishihara critically revised the manuscript.

\section{References}

1. Lutz W, Sanderson W, Scherbov S. The coming acceleration of global population ageing. Nature. 2008;451(7179):716-719.

2. Schafer I, von Leitner EC, Schon G, Koller D, Hansen H, Kolonko T, Kaduszkiewicz H, et al. Multimorbidity patterns in the elderly: a new approach of disease clustering identifies complex interrelations between chronic conditions. PLoS One. 2010;5(12):e15941.

3. Casselli G, Mesle F, Vallin J. Epidemiologic transition theory exceptions. Genus. 2002;58:9-51.

4. Harper K, Armelagos G. The changing disease-scape in the third epidemiological transition. Int J Environ Res Public Health. 2010;7(2):675-697.

5. From the Centers for Disease Control and Prevention. Public health and aging: trends in aging-United States and worldwide. JAMA. 2003;289(11):1371-1373.

6. Hajjar ER, Cafiero AC, Hanlon JT. Polypharmacy in elderly patients. Am J Geriatr Pharmacother. 2007;5(4):345351.

7. The Japan Geriatrics society. Guidelines for Medical Treatment and its Safety in the Elderly 2015. https://www. jpn-geriat-soc.or.jp/info/topics/pdf/20170808_01.pdf.

8. Farrell B, Shamji S, Monahan A, French Merkley V. Reducing polypharmacy in the elderly: Cases to help you "rock the boat". Can Pharm J (Ott). 2013;146(5):243244.

9. Zed PJ, Abu-Laban RB, Balen RM, Loewen PS, Hohl CM, Brubacher JR, Wilbur K, et al. Incidence, severity and preventability of medication-related visits to the emergency department: a prospective study. CMAJ. 2008;178(12):1563-1569.

10. Shah BM, Hajjar ER. Polypharmacy, adverse drug reactions, and geriatric syndromes. Clin Geriatr Med. 2012;28(2):173-186.

11. Drenth-van Maanen AC, van Marum RJ, Knol W, van der Linden CM, Jansen PA. Prescribing optimization method for improving prescribing in elderly patients receiving polypharmacy: results of application to case histories by general practitioners. Drugs Aging. 2009;26(8):687-701.

12. Maher RL, Hanlon J, Hajjar ER. Clinical consequences of polypharmacy in elderly. Expert Opin Drug Saf. 2014;13(1):57-65.

13. Doan J, Zakrzewski-Jakubiak H, Roy J, Turgeon J, Tannenbaum C. Prevalence and risk of potential cytochrome P450-mediated drug-drug interactions in older hospitalized patients with polypharmacy. Ann Pharmacother. 2013;47(3):324-332.

14. Hohl CM, Dankoff J, Colacone A, Afilalo M. Polypharmacy, adverse drug-related events, and potential adverse drug interactions in elderly patients presenting to an emergency department. Ann Emerg Med. 2001;38(6):666-671.

15. Kidney Disease: Improving Global Outcomes (KDIGO) CKD Work Group. KDIGO 2012 clinical practice guideline for the evaluation and management of chronic kidney 
disease. Kidney Int Suppl. 2013;3:1-150.

16. Kanda Y. Investigation of the freely available easy-touse software 'EZR' for medical statistics. Bone Marrow Transplant. 2013;48(3):452-458.

17. Silverberg D, Wexler D, Blum M, Wollman Y, Iaina A. The cardio-renal anemia syndrome: does it exist? Nephrol Dial Transplant. 2003;18 Suppl 8:7-12.

18. Sarnak MJ, Levey AS, Schoolwerth AC, Coresh J, Culleton B, Hamm LL, McCullough PA, et al. Kidney disease as a risk factor for development of cardiovascular disease: a statement from the American Heart Association Councils on Kidney in Cardiovascular Disease, High Blood Pressure Research, Clinical Cardiology, and Epidemiology and Prevention. Hypertension. 2003;42(5):1050-1065.

19. Horio T, Iwashima Y, Kamide K, Tokudome T, Yoshihara F, Nakamura S, Kawano Y. Chronic kidney disease as an independent risk factor for new-onset atrial fibrillation in hypertensive patients. J Hypertens. 2010;28(8):17381744.

20. Xu D, Murakoshi N, Sairenchi T, Irie F, Igarashi M, Nogami A, Tomizawa T, et al. Anemia and reduced kidney function as risk factors for new onset of atrial fibrillation (from the Ibaraki prefectural health study). Am J Cardiol. 2015;115(3):328-333.

21. McClellan WM, Flanders WD, Langston RD, Jurkovitz C, Presley R. Anemia and renal insufficiency are independ- ent risk factors for death among patients with congestive heart failure admitted to community hospitals: a population-based study. J Am Soc Nephrol. 2002;13(7):19281936.

22. Langston RD, Presley R, Flanders WD, McClellan WM. Renal insufficiency and anemia are independent risk factors for death among patients with acute myocardial infarction. Kidney Int. 2003;64(4):1398-1405.

23. Al-Ahmad A, Rand WM, Manjunath G, Konstam MA, Salem DN, Levey AS, Sarnak MJ. Reduced kidney function and anemia as risk factors for mortality in patients with left ventricular dysfunction. J Am Coll Cardiol. 2001;38(4):955-962.

24. Wexler D, Silverberg D, Blum M, Sheps D, Keren G, Wollman Y, Schwartz D, et al. Anaemia as a contributor to morbidity and mortality in congestive heart failure. Nephrol Dial Transplant. 2005;20(Suppl 7):11-15.

25. Groenveld HF, Januzzi JL, Damman K, van Wijngaarden J, Hillege HL, van Veldhuisen DJ, van der Meer P. Anemia and mortality in heart failure patients a systematic review and meta-analysis. J Am Coll Cardiol. 2008;52(10):818827.

26. Sico JJ, Concato J, Wells CK, Lo AC, Nadeau SE, Williams LS, Peixoto AJ, et al. Anemia is associated with poor outcomes in patients with less severe ischemic stroke. J Stroke Cerebrovasc Dis. 2013;22(3):271-278. 